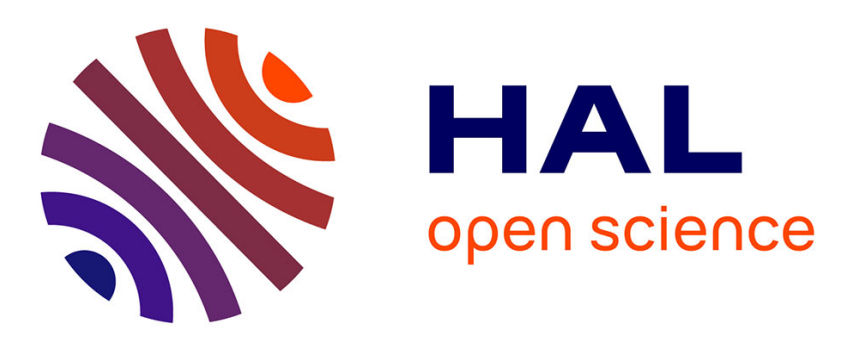

\title{
Online Parameter Estimation for Supercapacitor State-of-Energy and State-of-Health Determination in Vehicular Applications
}

Farshid Naseri, Ebrahim Farjah, Teymoor Ghanbari, Zahra Kazemi, Erik Schaltz, Jean-Luc Schanen

\section{To cite this version:}

Farshid Naseri, Ebrahim Farjah, Teymoor Ghanbari, Zahra Kazemi, Erik Schaltz, et al.. Online Parameter Estimation for Supercapacitor State-of-Energy and State-of-Health Determination in Vehicular Applications. IEEE Transactions on Industrial Electronics, 2020, 67 (9), pp.7963-7972. 10.1109/TIE.2019.2941151 . hal-03260244

\section{HAL Id: hal-03260244 \\ https://hal.science/hal-03260244}

Submitted on 14 Oct 2021

HAL is a multi-disciplinary open access archive for the deposit and dissemination of scientific research documents, whether they are published or not. The documents may come from teaching and research institutions in France or abroad, or from public or private research centers.
L'archive ouverte pluridisciplinaire HAL, est destinée au dépôt et à la diffusion de documents scientifiques de niveau recherche, publiés ou non, émanant des établissements d'enseignement et de recherche français ou étrangers, des laboratoires publics ou privés. 


\title{
Online Parameter Estimation for Supercapacitor State-of-Energy and State-of-Health Determination in Vehicular Applications
}

\author{
Farshid Naseri ，Student Member, IEEE, Ebrahim Farjah ，Member, IEEE, Teymoor Ghanbari ， \\ Zahra Kazemi ，Student Member, IEEE, Erik Schaltz ， and Jean-Luc Schanen
}

\begin{abstract}
Online accurate estimation of supercapacitor state-of-health (SoH) and state-of-energy (SoE) is essential to achieve efficient energy management and real-time condition monitoring in electric vehicle (EV) applications. In this article, for the first time, unscented Kalman filter (UKF) is used for online parameter and state estimation of the supercapacitor. In the proposed method, a nonlinear state-space model of the supercapacitor is developed, which takes the capacitance variation and self-discharge effects into account. The observability of the considered model is analytically confirmed using a graphical approach. The SoH and SoE are then estimated based on the supercapacitor online identified model with the designed UKF. The proposed method provides better estimation accuracy over Kalman filter (KF) and extended KF algorithms since the linearization errors during the filtering process are avoided. The effectiveness of the proposed approach is demonstrated through several experiments on a laboratory testbed. An overall estimation error below $0.5 \%$ is achieved with the proposed method. In addition, hardware-in-the-loop experiments are conducted and real-time feasibility of the proposed method is guaranteed.
\end{abstract}

Index Terms-Electric vehicles (EVs), state-of-energy (SoE), state-of-health (SoH), supercapacitor, unscented Kalman filter (UKF).

\section{INTRODUCTION}

$\mathbf{E}$ LECTRIC double-layer capacitors, also known as supercapacitors or ultracapacitors, have gained increasing attention from the transportation sector due to their appealing features

Manuscript received April 7, 2019; revised July 25, 2019; accepted August 25, 2019. Date of publication September 18, 2019; date of current version April 30, 2020. This work was supported in part by the French Ministry of Foreign Affairs and the Ministry of Research and Higher Education of France, and in part by the Iranian Ministry of Science Research and Technology, Center for International Scientific Studies and Cooperation (CISSC), within the frame of Gundishapur program. (Corresponding author: Ebrahim Farjah.)

F. Naseri, E. Farjah, T. Ghanbari, and Z. Kazemi are with the School of Electrical and Computer Engineering, Shiraz University, Shiraz 7134851154, Iran (e-mail: f.naseri@shirazu.ac.ir; farjah@shirazu.ac.ir; ghanbarih@shirazu.ac.ir; z.kazemi@shirazu.ac.ir).

E. Schaltz is with the Department of Energy Technology, Aalborg University, 9000 Aalborg, Denmark (e-mail: esc@et.aau.dk).

J.-L. Schanen is with the Grenoble Electrical Engineering Lab (G2ELab), 38031 Grenoble, France (e-mail: schanen@g2elab.inpg.fr).

Color versions of one or more of the figures in this article are available online at http://ieeexplore.ieee.org.

Digital Object Identifier 10.1109/TIE.2019.2941151 such as high power density, long cycle life, etc. In vehicular applications, the supercapacitor can be used as a complementary energy storage system (ESS) in conjunction with the chemical batteries to improve the vehicle performance during transient states such as acceleration and regenerative braking conditions [1]. For example, ENEA (Italian National Agency for New Technologies, Energy and Sustainable Economic Development) has recently developed an electric bus that actively combines the supercapacitors and batteries in a hybrid ESS, which shows the industrial importance of such systems [2]. However, the performance of the supercapacitor heavily depends on its stateof-health $(\mathrm{SoH})$ and state-of-energy (SoE). The $\mathrm{SoH}$ and $\mathrm{SoE}$ are critical metrics that determine how much energy the supercapacitor can absorb or release during a particular vehicle state [3]. Therefore, online accurate estimation of the foregoing variables is essential. The estimation accuracy of the $\mathrm{SoH}$ and $\mathrm{SoE}$ relies on the supercapacitor model fidelity and the estimation algorithm. In fact, an effective estimation method is needed for updating the model parameters in real-time to account for aging effects [4]. Different methods have been proposed for estimation of the supercapacitor SoH and SoE. In the following, a brief review of the state-of-the-art is presented.

The basic approach for the estimation of supercapacitor $\mathrm{SoH}$ is electrochemical impedance spectroscopy (EIS) [5], [6]. The EIS is a frequency-based characterization approach, which provides a very accurate estimation of SoH. However, the EIS requires costly instrumentation. More importantly, the EIS is an offline method and is not suitable for vehicular applications, where the estimation algorithm must run online. In [7], the $\mathrm{SoH}$ is estimated in an offline manner based on the bias voltage, current, and temperature during cycling tests. Artificial neural network has been used for SoH estimation in [8]. A frequency spectrometer has been used to obtain some training data in the frequency domain. The main drawback of this approach is that a sufficiently rich dataset is needed for the training phase, which makes its implementation difficult and time-consuming. In [9], the least-squares (LS) algorithm has been used for the estimation of supercapacitor states. However, the ordinary LS method is not suitable for online execution as its computational burden exponentially increases with the size of the measurement vector. To resolve the foregoing problem, recursive LS (RLS) algorithm has been used in [10]-[12] for state estimation in the supercapacitors. In [13], an online approach based on the extended RLS 
algorithm has been used to account for the undesirable effect of the measurement noises. However, all the foregoing LS-based approaches are designed based on the simple RC model of the supercapacitor, which neglects the self-discharging and charge redistribution effects.

In addition to the SoH, the supercapacitor SoE should also be accurately estimated. The basic approach for estimation of SoE is ampere-hour counting. However, the accuracy of this method is relatively low due to the accumulation of measurement errors over time. To rectify the foregoing problem, an effective SoE estimation approach based on the Luenberger style observer has been proposed in [14]. In this method, based on the difference between the actual and predicted supercapacitor voltages, a feedback loop is employed to compensate for the measurement errors, modeling uncertainties, and numerical computation errors. Another effective observer-based approach based on the generalized extended state observer (GESO) has been proposed for $\mathrm{SoH}$ and SoE estimation in [15]. The threebranch equivalent circuit model of the supercapacitor has been used together with the GESO. However, the effect of the leakage current is omitted in the estimation of SoE. It is noteworthy that the foregoing observer-based methods have relatively low computational burden since they fulfill the state estimation by only using the supercapacitor model. However, the observers are deterministic and thus, they cannot suitably deal with undesirable effects of the measurement noises with stochastic nature. Thus, in vehicular applications where the supercapacitor model parameters experience frequent variations and measurements are subjected to error and noise sources, a state estimation algorithm that has an inherent capability to deal with such modeling uncertainties, measurement errors, and stochastic noises is needed.

To tackle the foregoing problems, Kalman filter (KF) and extended KF (EKF)-based algorithms have been used for estimation of the supercapacitor states [16]-[18]. In [16] and [18], a three-branch equivalent circuit model of the supercapacitor is considered and KF has been used for estimation of the supercapacitor SoE. The model parameters and $\mathrm{SoH}$ are estimated with least mean square error (LMSE) fitting approach and KF is only used for estimation of the voltages across the capacitive branches. The main disadvantage of this approach is that the effect of the leakage current is ignored. Furthermore, the LMSE algorithm used for parameter estimation is not computationally efficient due to the involvement of a heavy matrix inversion process. In addition, the effects of measurement errors and noises cannot be suitably handled during parameter estimation with the LMSE algorithm. In [17], EKF has been used for estimation of the supercapacitor SoH. The EKF is used for estimation of the aging indicators using an RC equivalent circuit model, which takes into account the capacitance variation effect. At each iteration, the EKF considers a first-order linearization of the supercapacitor nonlinear model, which might lead to suboptimal performance and sometimes divergence of the filter. In addition, the considered model in [17] does not account for the self-discharging phenomenon of the supercapacitor.

Although there have been various studies for the estimation of supercapacitor SoH and SoE, there are still some points that remain to be addressed. In vehicular applications, where the real-time state estimation is must, a simplified supercapacitor model with relatively low computational burden is usually preferred. For instance, although the three-branch equivalent circuit model of the supercapacitor exhibits a very good accuracy, it necessitates the use of a separate algorithm for estimation of the supercapacitor unobservable internal state variables, which lowers the computational efficiency. On the other hand, the use of oversimplified supercapacitor models decreases the accuracy of the state estimation. Therefore, a supercapacitor model with moderate complexity can be useful if the state estimator has the inherent capability of effectively dealing with modeling uncertainties. More importantly, the state estimator should be able to handle the inherent sensor errors, sensor drift due to a change in the operating conditions, errors associated with analog to digital conversion (ADC) units, electromagnetic interference, noise effects, etc. As discussed before, the KF-based filtering methods are best suited for dealing with the stochastic nature of the modeling uncertainties and measurement errors. The filter-based techniques have also been used for state estimation in other ESS types such as electrochemical batteries, which indicates the usefulness if these estimation tools [19], [20]. However, the KF and EKF use a linearized supercapacitor model, which decreases the accuracy of the state estimation. In addition, the EKF algorithm has high computational burden since a Jacobian matrix needs to be calculated at each iteration of the algorithm.

To address the mentioned issues, in this article, for the first time, unscented KF (UKF) algorithm is used for accurate concurrent estimation of the supercapacitor SoH and SoE. An RC equivalent circuit model of the supercapacitor, which effectively takes into account the capacitor variation and selfdischarging effects, is considered in the UKF algorithm. Unlike other approaches that fulfill the parameter and state estimation in separate steps, the proposed UKF-based approach obtains the supercapacitor SoH and SoE using only one filtering process. The foregoing technique increases the estimation accuracy by taking into account the cross-correlations between the parameters and states. In addition, it has easier implementation since only one filtering algorithm is needed. The main features of the proposed approach are highlighted as follows.

1) This article is the first attempt for using UKF in joint estimation of the supercapacitor SoH and SoE. The UKF provides better accuracy over existing methods such as EKF but remarkably, the computational complexity of the UKF is lower than the EKF, as will be proved later.

2) The capacitance variation and self-discharging effects are considered in the supercapacitor model.

3) Both the SoH and SoE are estimated using a single UKFbased filtering process, which is accomplished by augmenting the internal voltage of the supercapacitor as a new state variable with the main system model.

4) The real-time feasibility of the proposed UKF-based approach is demonstrated by a series of hardware-in-theloop (HIL) experiments.

The rest of the article is structured as follows. In Section II, the operating principles of the proposed method are presented. In Section III, the experimental results of the proposed approach on 
an implemented testbed are provided and discussed. To demonstrate the real-time feasibility of the proposed method, some HIL experiments are conducted and the results are reported in Section IV. In Section V, the proposed approach is compared with the KF-based and EKF-based algorithms in terms of accuracy and computational complexity. Finally, Section VI concludes this article. An Appendix provides some details regarding the implemented algorithm.

\section{Operating Principles of the Proposed Method}

The key step for accurate estimation of the SoH and SoE lies in the precise parameter estimation and successful observation of the supercapacitor internal voltage. In this article, both the parameter and internal state estimation tasks are fulfilled using the proposed UKF-based approach. Different steps of the proposed method are explained in the following subsections.

\section{A. Problem Statement}

The target of this article is to develop an online, accurate, and computationally friendly $\mathrm{SoH}$ and $\mathrm{SoE}$ indicator. It is assumed that the supercapacitor measurable parameters are its terminal voltage and charge/discharge current. The equivalent series resistance (ESR) $R_{s}$ and the internal capacitance $C$ are considered as the key signatures for indicating the supercapacitor $\mathrm{SoH}$. According to the estimates of the foregoing parameters, the supercapacitor SoH can then be quantified based on an end-of-life (EoL) criterion. For example, according to IEC-62391, the supercapacitor reaches its EoL when the ESR increases by two times of the rated ESR. Therefore, the SoH can be calculated as follows:

$$
\mathrm{SoH}(\%)=\frac{2 \mathrm{ESR}_{\text {rated }}-\mathrm{ESR}_{\text {estimated }}}{\mathrm{ESR}_{\text {rated }}} \times 100
$$

where $\mathrm{ESR}_{\text {rated }}$ and $\mathrm{ESR}_{\text {estimated }}$ are the rated and estimated ESR values, respectively. In addition, SoE is defined as the remaining energy, which is shown in percentage. The stored energy of the supercapacitor can be given by

$$
\begin{aligned}
E & =\int Q d \hat{v}_{c}=\int C \hat{v}_{c} d \hat{v}_{c}=\int C_{0} \hat{v}_{c} d \hat{v}_{c}+\int C_{1} \hat{v}_{c}^{2} d \hat{v}_{c} \\
& =\frac{1}{2} C_{0} \hat{v}_{c}^{2}+\frac{1}{3} C_{1} \hat{v}_{c}^{3}
\end{aligned}
$$

where $E$ is the stored energy in Joules, $Q$ is the electric charge, $C$ is the internal capacitance, and $\hat{v}_{c}$ is the estimated internal voltage of the supercapacitor. The SoE is calculated as the ratio of the remaining energy to the maximum energy of the supercapacitor $E_{\max }$ in percentage:

$$
\operatorname{SoE}(\%)=\frac{E}{E_{\max }} \times 100 \text {. }
$$

The maximum energy $E_{\max }$ is derived when the supercapacitor internal voltage $v_{c}$ equals to the rated supercapacitor voltage $V_{\text {rated. }}$ Therefore, the accurate estimation of SoH and SoE relies on the precise estimation of the supercapacitor internal voltage and its model parameters.

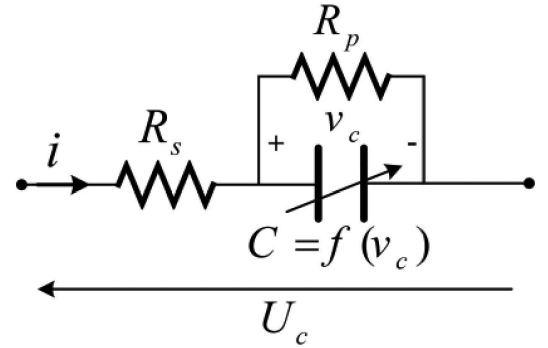

Fig. 1. RC equivalent circuit model of the supercapacitor considering the self-discharge and capacitance variation effects.

\section{B. Supercapacitor Nonlinear State-Space Model}

In this article, a first-order equivalent circuit model of the supercapacitor is used. In order to effectively mimic the supercapacitor real behavior, the capacitance variation and charge redistribution effects are taken into account. The considered model is shown in Fig. 1, in which $U_{c}, i$, and $v_{c}$ are the terminal voltage, current, and supercapacitor internal voltage, respectively. In addition, $R_{s}$ is the $\mathrm{ESR}, R_{p}$ is the equivalent parallel resistance, and $C$ is the supercapacitor voltage-dependent capacitance, which is described with the following expression [21], [22]:

$$
C=g\left(v_{c}\right)=C_{0}+C_{1} v_{c}
$$

In (4), $C_{0}$ is a constant capacitance. In addition, it can be assumed that the capacitance $C$ linearly evolves with an almost constant or a slow time-varying slope and thus, $d C_{1} / d t \approx 0$. The voltage across the supercapacitor internal capacitance can be written as

$$
v_{c}=\frac{1}{C} \int\left(i-\frac{v_{c}}{R_{p}}\right) d t
$$

The supercapacitor internal voltage $v_{c}$ is considered as the first state variable $\left(x_{1}=v_{c}\right)$. Taking the derivative of (5), one can write

$$
\begin{aligned}
\frac{d v_{c}}{d t} & =\frac{d}{d t}\left(\frac{1}{C}\right) \int\left(i-\frac{v_{c}}{R_{p}}\right) d t+\frac{1}{C}\left(i-\frac{v_{c}}{R_{p}}\right) \\
& =\frac{d}{d t}\left(\frac{1}{C}\right) \int i d t-\frac{1}{R_{p}} \frac{d}{d t}\left(\frac{1}{C}\right) \int v_{c} d t+\frac{1}{C}\left(i-\frac{v_{c}}{R_{p}}\right) .
\end{aligned}
$$

The integration of $v_{c}$ is also considered as a second state variable $\left(x_{2}=\int v_{c} d t\right)$. From (4) and (6), $d C / d t$ can be written as follows:

$$
\begin{aligned}
\frac{d C}{d t} & =C_{1} \frac{d v_{c}}{d t} \\
& =C_{1}\left(\frac{d}{d t}\left(\frac{1}{C}\right) \int i d t-\frac{1}{R_{p}} \frac{d}{d t}\left(\frac{1}{C}\right) x_{2}+\frac{1}{C}\left(i-\frac{x_{1}}{R_{p}}\right)\right) .
\end{aligned}
$$


Considering $1 / C$ as a state variable and using (7), the derivative of $1 / C$ can be obtained as follows:

$$
\begin{aligned}
\frac{d}{d t}\left(\frac{1}{C}\right)= & \frac{-1}{C^{2}} \frac{d C}{d t} \\
= & \frac{-1}{C^{2}} C_{1}\left(\frac{d}{d t}\left(\frac{1}{C}\right) \int i d t-\frac{1}{R_{p}} \frac{d}{d t}\left(\frac{1}{C}\right) x_{2}\right. \\
& \left.+\frac{1}{C}\left(i-\frac{x_{1}}{R_{p}}\right)\right) \\
= & \frac{-C_{1}}{C^{2}} \frac{d}{d t}\left(\frac{1}{C}\right) \int i d t+\frac{C_{1} x_{2}}{C^{2} R_{p}} \frac{d}{d t}\left(\frac{1}{C}\right) \\
& -\frac{C_{1}}{C^{3}}\left(i-\frac{x_{1}}{R_{p}}\right) .
\end{aligned}
$$

With some manipulations, the following state equation can be derived:

$$
\begin{gathered}
\frac{d}{d t}\left(\frac{1}{C}\right) \underbrace{\left[1+\frac{C_{1}}{C^{2}} \int i d t-\frac{C_{1} x_{2}}{C^{2} R_{p}}\right]}_{A}=-\frac{C_{1}}{C^{3}}\left(i-\frac{x_{1}}{R_{p}}\right) \\
\Rightarrow \frac{d}{d t}\left(\frac{1}{C}\right)=\frac{1}{A} \frac{-C_{1}}{C^{3}}\left(i-\frac{x_{1}}{R_{p}}\right) .
\end{gathered}
$$

The supercapacitor voltage $U_{c}$ is considered as the system output $y$ as follows:

$$
y=U_{c}=R_{s} i+v_{c}
$$

where the supercapacitor current $i$ is considered as the system input $u$. The state vector is then considered as follows:

$$
X=\left[\begin{array}{lllllll}
x_{1} & x_{2} & x_{3} & x_{4} & x_{5} & x_{6} & x_{7}
\end{array}\right]^{T}=\left[\begin{array}{lll}
v_{c} & v_{c} R_{s} R_{p} C_{1} \frac{1}{C} y
\end{array}\right]^{T} .
$$

In (11), the parameters $R_{s}, R_{p}$, and $C_{1}$ are also considered as state variables and since these parameters have a slow timevarying nature, the following state equations can be deducted:

$$
\begin{aligned}
& x_{3}=R_{s} \rightarrow \dot{x}_{3}=0, x_{4}=R_{p} \rightarrow \dot{x}_{4}=0 \\
& x_{5}=C_{1} \rightarrow \dot{x}_{5}=0 .
\end{aligned}
$$

Considering (4)-(9), other state-space equations can be written as follows:

$$
\begin{aligned}
\dot{x}_{1}= & \left(\frac{-1}{1+x_{5} x_{6}^{2} \int u d t-\left(x_{6}^{2} x_{5} x_{2}\right) / x_{4}}\right) x_{5} x_{6}^{2}\left(u-\frac{x_{1}}{x_{4}}\right) \\
& \times\left(\int u d t-\frac{x_{2}}{x_{4}}\right)+x_{6}\left(u-\frac{x_{1}}{x_{4}}\right) \\
\dot{x}_{2}= & x_{1} \\
\dot{x}_{6}= & \left(\frac{-1}{1+x_{5} x_{6}^{2} \int u d t-\left(x_{6}^{2} x_{5} x_{2}\right) / x_{4}}\right) x_{5} x_{6}^{3}\left(u-\frac{x_{1}}{x_{4}}\right) .
\end{aligned}
$$

As seen in (11), the system output $\left(y=U_{c}\right)$ is also considered as a state variable. In the next subsection, it is explained that $x_{7}=y$ is considered to ensure the model observability in all

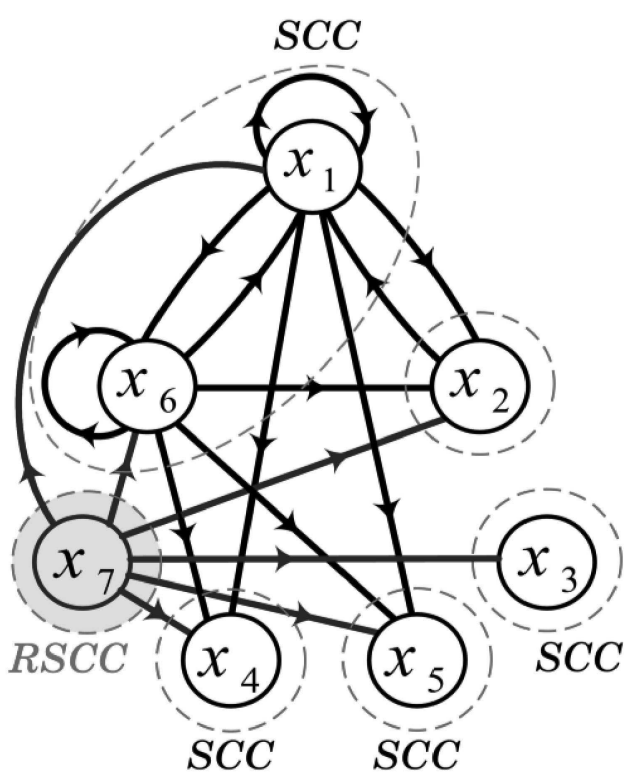

Fig. 2. Inference diagram of the supercapacitor internal states. The sensory node indicated with blue color is related to the supercapacitor terminal voltage $U_{c}$. Balance equations of $x_{1}-x_{7}$ are represented by (12)-(16).

operating conditions. Hence, the last state-space equation can be derived as follows:

$$
\begin{aligned}
x_{7} & =y \stackrel{\operatorname{From}(7)}{\longrightarrow} \dot{x}_{7}=\dot{y}=\dot{x}_{3} u+x_{3} \dot{u}+\dot{x}_{1} \\
& =x_{3} \dot{u}+\dot{x}_{1}
\end{aligned}
$$

where $\dot{x}_{1}$ should be substituted from (13).

\section{Observability of the System}

In this article, the system observability is demonstrated using an innovative graphical approach recently proposed by Liu et al. [23]. In this approach, the dynamic interdependence between the system states will be exploited through a so-called inference diagram. The system inference diagram is obtained through the following steps:

1) If $x_{j}$ appears in the differential equation of $x_{i}$, a direct link $x_{i} \rightarrow x_{j}$ is drawn, which implies that the information on $x_{j}$ can be collected by monitoring $x_{i}$ as a function of time. The inference diagram for the considered system is shown in Fig. 2.

2) The obtained inference diagram is then decomposed into a unique set of maximal strongly connected components (SCCs). The SCCs are the largest subgraphs selected such that there is a straight path from each node to all other nodes in that subgraph. The SCCs are surrounded by the red dashed circles in Fig. 2.

3) The SCCs that have no incoming edges are defined as root SCCs (RSCCs).

Definition 1: The necessary and sufficient condition for observability of all system states is that in the inference diagram, at least one node from each RSCC is a sensory node [23]. 
As seen in Fig. 2, only one RSCC exists in the system inference diagram. The RSCC includes the state variable $x_{7}$, which is equal to the supercapacitor measured terminal voltage. Therefore, since RSCC includes a sensory node, based on Definition 1 , the observability of the whole system can be guaranteed [23].

In this article, the supercapacitor model consisting of (12)(16) are used for supercapacitor $\mathrm{SoH}$ and SoE estimation and sufficiently good results are obtained. However, more complicated supercapacitor models such as the three-branch equivalent circuit model can also be used with the proposed UKF-based approach to further improve the estimation accuracy.

\section{Proposed UKF-Based SoH and SoE Indicator}

The UKF algorithm has been widely used for state estimation. Unlike the EKF, which involves a linearization stage through the calculation of a Jacobian matrix (partial derivative matrices), the UKF has less computational effort as it does not depend on Jacobians [24]-[26]. In a general case, the discrete-time state-space representation of the supercapacitor model can be expressed as follows:

$$
\begin{aligned}
X_{k+1} & =f\left(X_{k}, u_{k}, t_{k}\right)+\omega_{k} \\
y_{k} & =h\left(X_{k}, t_{k}\right)+\nu_{k} \\
\omega_{k} & \sim\left(0, Q_{k}\right) \\
\nu_{k} & \sim\left(0, R_{k}\right)
\end{aligned}
$$

where $X_{k}$ is the state vector at sample $k, u$ is the system input (supercapacitor current), $\omega$ is the process noise, and $\nu$ is the measurement noise. The process and measurement noises are considered to be uncorrelated Gaussian white noises, which are included to account for the modeling uncertainties and measurement errors. In the first two formulas of (17), $f(\cdot)$ and $h(\cdot)$ are nonlinear functions, which express alternative representations of the system model (12)-(16). The third and fourth formulas in (17) show that the process and measurement noises have zero mean and covariance matrices $Q^{7 \times 7}$ and $R^{1 \times 1}$, respectively. At the first step, the UKF algorithm is initialized by assigning initial values to the system states $\left(\hat{X}_{0}^{+}\right)$and the covariance matrix of the estimation error $\left(P_{0}^{+}\right)$. The covariance matrix $P$ exhibits the uncertainty in the estimated system states. The initializing process is only fulfilled at the first iteration $(k=1)$. The UKF algorithm performs a nonlinear transformation (unscented transform) on a series of the so-called sigma points in state space whose probability density function (PDF) suitably approximates the true PDF of the state vector. In the considered supercapacitor model, there exist $m=7$ state variables and thus, $2 m=14$ different sigma points are selected as follows [27]:

$$
\begin{gathered}
\tilde{X}^{(i)}=\left(\sqrt{7 P_{k-1}^{+}}\right)_{i}^{T}, i=1,2, \ldots, 7 \\
\tilde{X}^{(i+7)}=-\left(\sqrt{7 P_{k-1}^{+}}\right)_{i}^{T}, i=1,2, \ldots, 7 \\
\hat{X}_{k-1}^{(i)}=\hat{X}_{k-1}^{+}+\tilde{X}^{(i)}, i=1,2, \ldots, 14 .
\end{gathered}
$$

Then, the known nonlinear supercapacitor model $f(\cdot)$ is used to transform the sigma points into $\hat{X}_{k}^{(i)}$ vectors as follows:

$$
\hat{X}_{k}^{(i)}=f\left(\hat{X}_{k-1}^{(i)}, u_{k}, t_{k}\right) .
$$

The time update phase for obtaining the a priori state estimates and the covariance matrix of the estimation error is fulfilled using the following expressions:

$$
\begin{aligned}
& \hat{X}_{k}^{-}=\frac{1}{14} \sum_{i=1}^{14} \hat{X}_{k}^{(i)} \\
& P_{k}^{-}=\frac{1}{14} \sum_{i=1}^{14}\left(\hat{X}_{k}^{(i)}-\hat{X}_{k}^{-}\right)\left(\hat{X}_{k}^{(i)}-\hat{X}_{k}^{-}\right)^{T}+Q_{k-1}
\end{aligned}
$$

where $\hat{X}_{k}^{-}$is the priori estimation up to the sample $k$. Next, the measurement update phase is fulfilled considering a new set of sigma points as follows:

$$
\begin{gathered}
\tilde{X}^{(i)}=\left(\sqrt{7 P_{k}^{-1}}\right)_{i}^{T}, i=1,2, \ldots, 7 \\
\tilde{X}^{(i+7)}=-\left(\sqrt{7 P_{k}^{-1}}\right)_{i}^{T}, i=1,2, \ldots, 7 \\
\hat{X}_{k}^{(i)}=\hat{X}_{k}^{-}+\tilde{X}^{(i)}, i=1,2, \ldots, 7 .
\end{gathered}
$$

It should be noted that the same sigma points of (18) (from the time update phase) can be reused during the measurement update phase to further save the computational effort, which is of great importance for real-time vehicular applications [27]. The known nonlinear output equation $h(\cdot)$ is subsequently used to transform the sigma points (21) into $\hat{y}_{k}^{(i)}$ vectors as follows:

$$
\hat{y}_{k}^{(i)}=h\left(\hat{X}_{k}^{(i)}, t_{k}\right) .
$$

In order to calculate the predicted measurement and its covariance matrix at time $k$, the following formulas are used:

$$
\begin{aligned}
& \hat{y}_{k}=\frac{1}{14} \sum_{i=1}^{14} \hat{y}_{k}^{(i)} \\
& P_{y}=\frac{1}{14} \sum_{i=1}^{14}\left(\hat{y}_{k}-\hat{y}_{k}^{(i)}\right)\left(\hat{y}_{k}-\hat{y}_{k}^{(i)}\right)^{T}+R_{k} .
\end{aligned}
$$

Note that $R_{k}$ is added in the second formula of (23) to account for the effect of measurement noise. The cross-covariance between $\hat{y}_{k}$ and $\hat{X}_{k}^{-}$can also be obtained as follows:

$$
P_{x y}=\frac{1}{14} \sum_{i=1}^{14}\left(\hat{X}_{k}^{(i)}-\hat{X}_{k}^{-}\right)\left(\hat{y}_{k}^{(i)}-\hat{y}_{k}\right)^{T} .
$$

Finally, the measurement at instant $k$ is taken into account during the measurement update step as follows:

$$
\begin{aligned}
K_{k}(\text { Kalman Gain }) & =P_{x y} P_{y}^{-1} \\
\hat{X}_{k}^{+} & =\hat{X}_{k}^{-}+K_{k}\left(y_{k}-\hat{y}_{k}\right) \\
P_{k}^{+} & =P_{k}^{-}-K_{k} P_{y} K_{k}^{T} .
\end{aligned}
$$



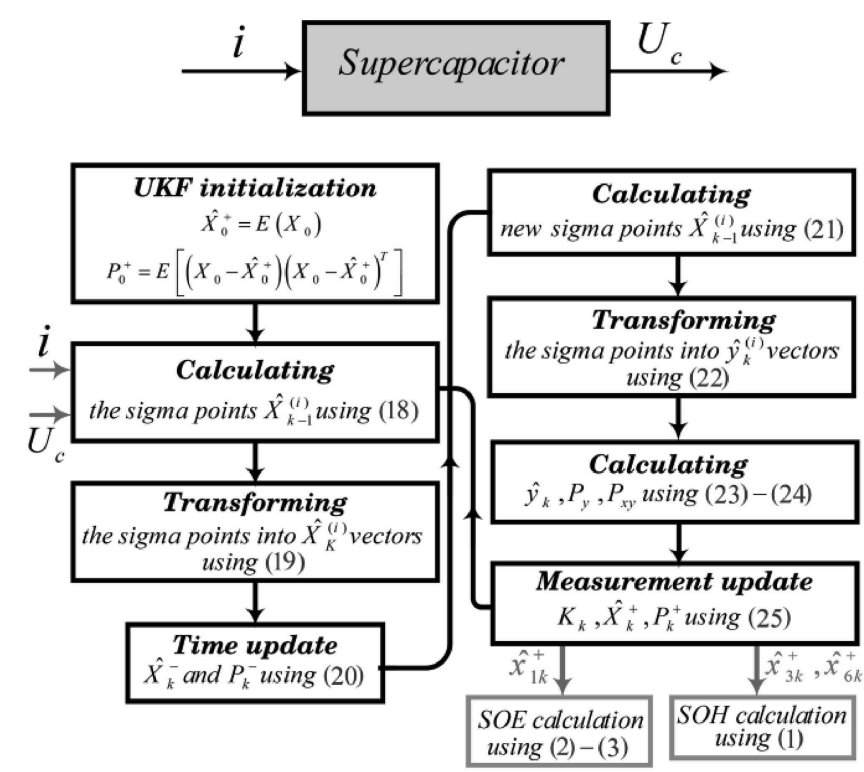

Fig. 3. Flowchart of the proposed UKF-based SoE and SoH estimation approach.

The second formula of (25) gives the final state estimates, in which $\hat{x}_{3 k}^{+}$and $\hat{x}_{6 k}^{+}$are the supercapacitor $\mathrm{SoH}$ indicators. In addition, $\hat{x}_{1 k}^{+}$can be used to calculate the SoE using (2), (3). The whole algorithm flow of the proposed approach is illustrated in Fig. 3.

\section{E. Settings of the Proposed UKF-Based Approach}

As explained before, the state estimator must be initialized at the first iteration $(k=1)$. In order to demonstrate the merits of the proposed approach more intuitively, it is herein assumed that no initial information about the system states is available. Therefore, the initial values of $x_{1}-x_{7}$ and the covariance matrix of the estimation error are set as follows:

$$
\begin{aligned}
& \hat{X}_{0}^{+}=0^{7 \times 1} \\
& P_{0}^{+}=10 \times I^{7 \times 7}
\end{aligned}
$$

where 0 and $I$ are zero and identity matrices, respectively. However, in order to increase the filter convergence speed, the initial values of the state variables can be set to their rated values, most of which are known from the supercapacitor datasheet or by a standard offline test. The covariance matrices of the process and measurement noises reflect the accuracy levels of the sensor measurements as well as the considered supercapacitor model. The covariance matrix of the measurement noise is chosen based on the typical errors of the voltage sensor as well as the ADC units. In addition, the diagonal covariance matrix of the process noise is selected with trial and error. These matrices are assigned as follows:

$$
R=0.015, Q=\operatorname{diag}(0.1,0.1,0.1,0.5,0.3,0.1,0.2) .
$$

More theoretic information about the optimal selection of the covariance matrices of the measurement noise and process noise can be found in [27].

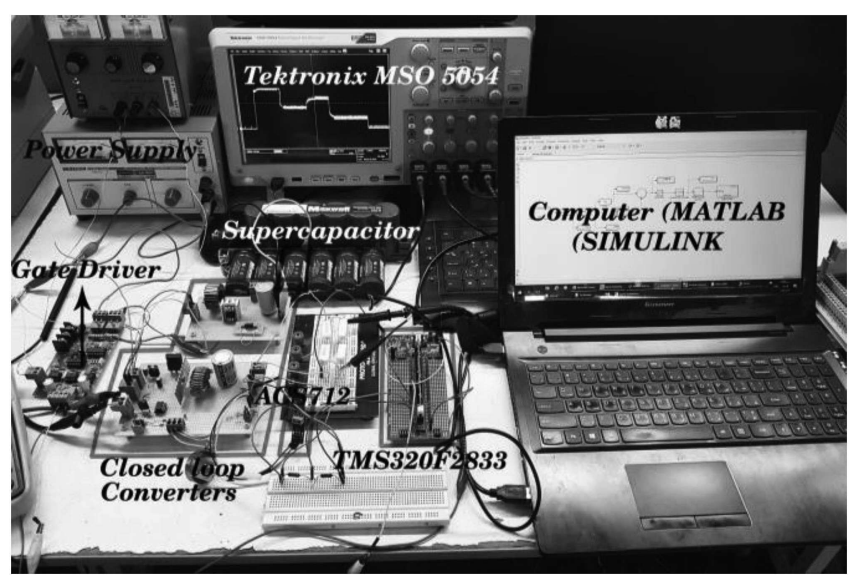

Fig. 4. Experimental testbench for testing the proposed UKF-based approach.

\section{Experimental REsults}

In order to assess the performance of the proposed method, several experiments are conducted and the results are presented in this section. In the following, the experimental testbed is introduced and the results are discussed in details.

\section{A. Description of the Implemented Testbed}

The experimental testbench is shown in Fig. 4. A supercapacitor cell (from Maxwell Technologies) with rated voltage and capacitance of $2.7 \mathrm{~V}$ and $350 \mathrm{~F}$, respectively, is used in the experiments. Detailed information about the parameters of the utilized supercapacitor cell is provided in the Appendix. In order to generate the desired current profiles and to emulate the real-life electric vehicle (EV) driving conditions, a closed-loop buck converter and a dc electronic load are implemented. The closed-loop buck converter controls the supercapacitor charge current. Likewise, the dc electronic load controls the discharge current of the supercapacitor. The dc electronic load is realized by closed-loop control of the gate-source voltage of a linear MOSFET (IXTK90N25L2), which is cascaded with a resistive load. The digital signal processor (DSP) TMS320F28335 is used for closed-loop control of the converters. The PI controllers for current regulation and the algorithm of the proposed UKF-based approach for estimation of the supercapacitor $\mathrm{SoH}$ and $\mathrm{SoE}$ are realized in MATLAB/Simulink. The experiments are performed at room temperature $\left(T=25^{\circ} \mathrm{C}\right)$. The ACS712ELCTR$20 \mathrm{~A}-\mathrm{T}$ current sensor is used for measuring the supercapacitor charge/discharge current. In addition, the supercapacitor voltage is directly read by 12-bits ADC unit of the microprocessor. A sampling frequency of $f_{s}=1 \mathrm{kHz}$ is selected. A complete list of the experimental parameters is provided in Table I.

\section{B. Results and Discussions}

In order to test the proposed UKF-based method, a number of scenarios are considered. Based on the charging/discharging current profiles that may occur in a real EV drive cycle, three scenarios are examined as follows. 
TABLE I

EXPERIMENTAL PARAMETERS OF THE TEST SyStEM

\begin{tabular}{cc}
\hline Parameter & Value \\
\hline \hline Supercapacitor cell & Maxwell D-Cell® \\
Rated voltage of cell & $2.7 \mathrm{~V}$ \\
Rated capacitance of cell & $350 \mathrm{~F}$ \\
Current sensor & ACS712ELCTR-20A-T \\
Controller unit & TMS320F28335 \\
Sampling frequency $f_{s}$ & $1 \mathrm{kHz}$ \\
Operating temperature $T$ & $25^{\circ} \mathrm{C}$ \\
\hline
\end{tabular}

Case A: In this case, the initial SoE of the supercapacitor is set to zero $\left(\mathrm{SoE}_{\text {initial }}=0\right)$ and the supercapacitor gets charged with a constant current $i=-2.5 \mathrm{~A}$. This case simulates the regenerative braking or EV coasting.

Case B: In this case, the initial $\mathrm{SoE}$ of the supercapacitor is set to $90 \%\left(\operatorname{SoE}_{\text {initial }}=90 \%\right)$ and the supercapacitor gets discharged with a constant current $i=+2.5 \mathrm{~A}$. This case emulates the vehicle acceleration mode, in which the supercapacitor gets discharged to support the main energy storage unit.

Case C: In this case, the initial SoE is set to $50 \%$ $\left(\mathrm{SoE}_{\text {initial }}=50 \%\right)$. The supercapacitor is first discharged with $i=+2.5 \mathrm{~A}$ and then, it is charged with $i=-2.5 \mathrm{~A}$ followed by a rest condition for $\Delta t=50 \mathrm{~s}$. The pattern is repeated twice during the experimentation time $\Delta t=600 \mathrm{~s}$.

The charging/discharging current profiles in Cases A-C are depicted in Fig. 5. In Case A, the charging process is stopped when the cell is fully charged to $2.7 \mathrm{~V}$. Likewise, in Case B, the discharge current is set to zero (the load is disconnected) when the cell is fully discharged. To assess the robustness of the proposed approach against the measurement noises and errors, a fourth case (Case D) is also considered which is similar to Case A except that band-limited white noise with a signal to noise ratio (SNR) of $30 \mathrm{~dB}$ is added to the current and voltage measurements. In order to assess the accuracy of the proposed UKF-based estimator, the EIS method is used as a benchmark. The obtained parameters of the supercapacitor model are as follows:

$$
\left[\begin{array}{llll}
R_{s} & R_{p} & C_{1} & C_{0}
\end{array}\right]^{T}=\left[\begin{array}{lllll}
3.3 \mathrm{~m} \Omega & 10 \mathrm{k} \Omega & 0.91348 \mathrm{~F}
\end{array}\right]^{T}
$$

The supercapacitor states $R_{s}$ and $C$ reflect the SoH. The estimation accuracy of these parameters is assessed using the following formula:

$$
\text { SoH indicators errors } \%=\frac{x_{\text {estimated }}-x_{\text {EIS }}}{x_{\text {EIS }}} \times 100
$$

where $x$ is either of the SoH indicators $\left(R_{s}\right.$ or $\left.C\right), x_{\text {estimated }}$ is the estimated value of the state, and $x_{\text {EIS }}$ is the real value of the system state obtained using the EIS. Furthermore, the estimation accuracy of SoE is calculated using the following formula:

$$
\begin{aligned}
\text { SoE error } \% & =\frac{\text { SoE }_{\text {estimated }}-\mathrm{SoE}_{\text {real }}}{\mathrm{SoE}_{\text {real }}} \times 100 \\
& =\frac{E_{\text {estimated }}-E_{\text {real }}}{E_{\text {real }}} \times 100
\end{aligned}
$$

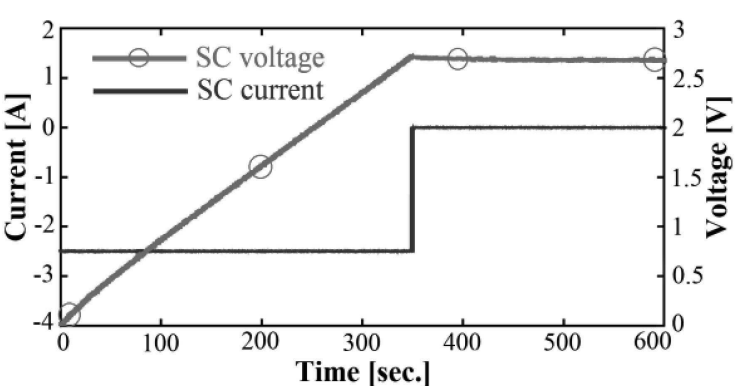

(a)

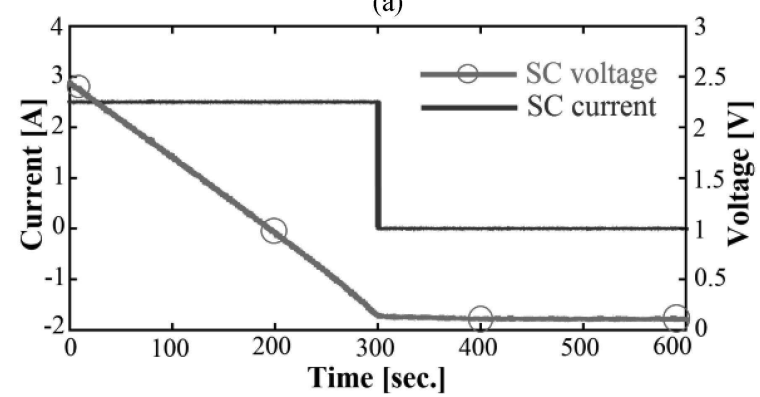

(b)

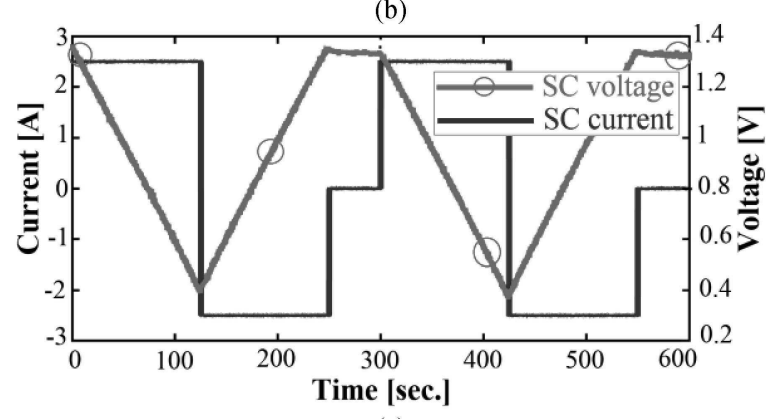

(c)

Fig. 5. Current and voltage profiles of the supercapacitor in scenarios A-C. (a) Scenario A. (b) Scenario B. (c) Scenario C.

where $S_{0} E_{\text {estimated }}$ and $E_{\text {estimated }}$ are the estimated SoE and estimated supercapacitor remaining energy, respectively. In addition, $\mathrm{SoE}_{\text {real }}$ and $E_{\text {real }}$ are the real $\mathrm{SoE}$ and remaining energy of the supercapacitor, respectively. $E_{\text {real }}$ is obtained as follows:

$$
E_{\text {real }}=E_{0}+\underbrace{\int\left(i \times U_{c}\right) d t}_{\text {input energy }}-\underbrace{\left(\int R_{s} i^{2} d t+\int \frac{v_{c}^{2}}{R_{p}} d t\right)}_{\text {energy loss }}
$$

where $E_{0}$ is the initial stored energy of the supercapacitor. The state estimation results for Cases A, B, and D are shown in Fig. 6. Due to the space limit, only the results related to $x_{3}-x_{6}$ are given. As seen, under no circumstance, the maximum convergence time of the proposed UKF-based estimator exceeds $1.5 \mathrm{~s}$. In addition, the average error [calculated using (26) over a time period of $5 \mathrm{~s}$ after the convergence of the filter] for estimating the $\mathrm{SoH}$ indicators $R_{s}$ and $C$ are $\approx 0.52 \%$ and $\approx 0.32 \%$, respectively. It can be seen that even when the measurements are contaminated with random noise with SNR of up to $30 \mathrm{~dB}$, the $\mathrm{SoH}$ indicators are accurately estimated. The faster convergence time in Case A is obtained since the selected initial SoE in Case A is closer to the real initial state vector in the UKF algorithm. The estimated $\mathrm{SoH}$ indicators in Case $\mathrm{C}$ are also 


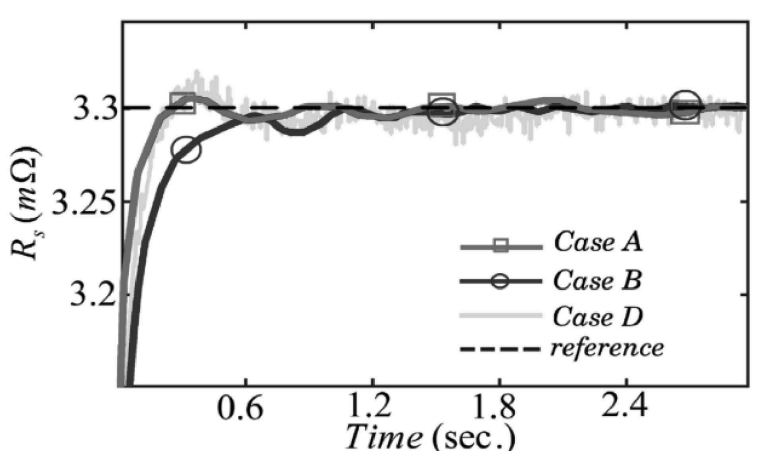

(a)

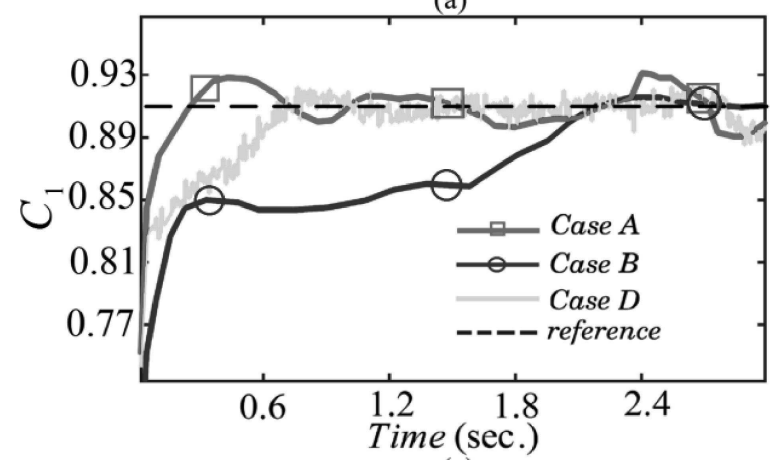

(c)

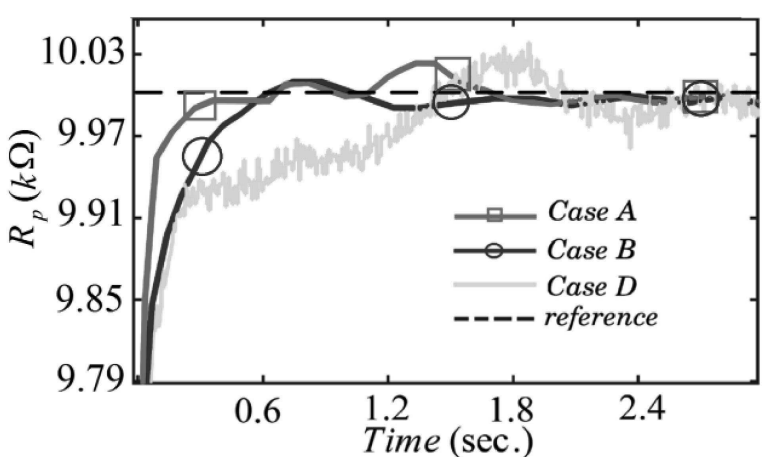

(b)

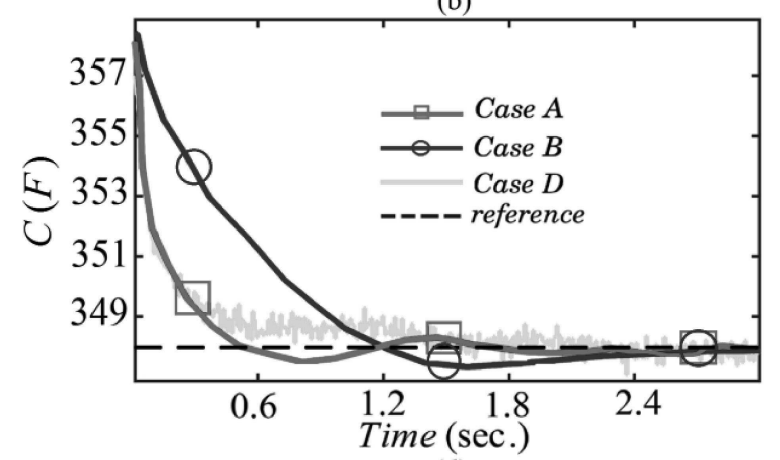

(d)

Fig. 6. State estimation results with the proposed UKF-based approach for cases A, B, and D. (a) Estimation results for $R_{s}$. (b) Estimation results for $R_{p}$. (c) Estimation results for $C_{1}$. (d) Estimation results for $C$.

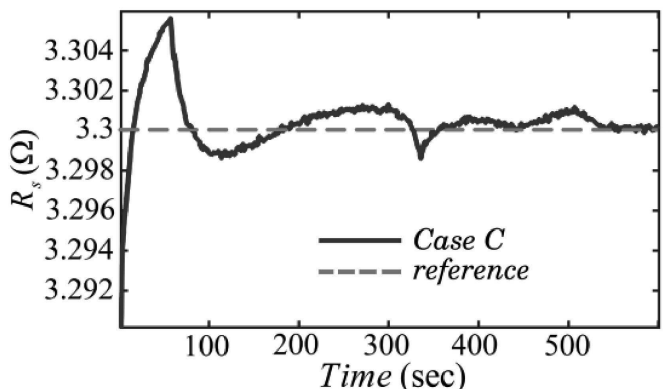

(a)

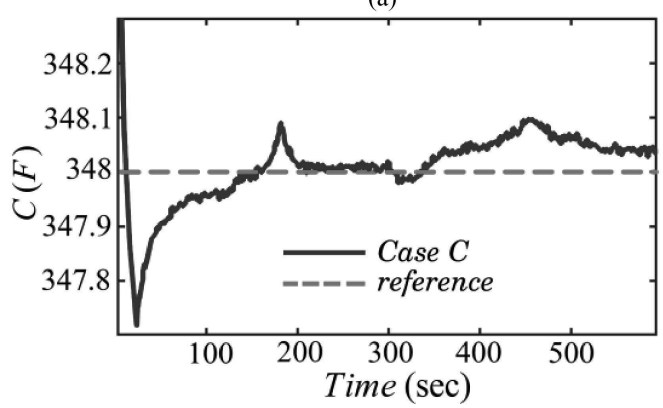

(b)

Fig. 7. Estimated $\mathrm{SoH}$ indicators with the proposed approach in Case C.

shown in Fig. 7. It can be deduced that the proposed estimator effectively estimates the supercapacitor parameters in different charging/discharging modes.

In Fig. 8, the results of the SoE estimation in Cases A, B, and $\mathrm{C}$ are depicted. It can be observed that in all Cases, the
SoE is accurately estimated. At the beginning stages of Cases $\mathrm{B}$ and $\mathrm{C}$, relatively large differences between the estimated and real SoE are observable, which is due to the fact that the initial SoE of the supercapacitor in Cases B and C is set to $90 \%$ and $50 \%$, respectively, which is different from the considered initial SoE of $0 \%$ in the UKF algorithm. However, it is seen that the SoE successfully converges to its reference value in a very short duration. The results also reveal that during the rest periods when the supercapacitor charging/discharging is terminated, the SoE gradually decreases due to the self-discharge effect caused by the parallel resistance in the supercapacitor model. The state estimation errors of the supercapacitor SoE in Cases A-D are also calculated using (27) and (28) and are summarized in Table II, which reports the mean error values over the simulation time. The results show that the error of the SoE estimation does not exceed $1 \%$ in the worst case when the measurement data are contaminated with random noise with SNR of up to $30 \mathrm{~dB}$.

\section{REAL-Time Feasibility DEMONStration With HIL EXPERIMENTS}

In order to demonstrate the real-time feasibility of the proposed UKF-based approach, a series of HIL experiments are conducted. The photo of the HL test is shown in Fig. 9. The proposed UKF-based method is completely implemented in MATLAB/Simulink environment. Therefore, the $\mathrm{C}$ code of the algorithm is first generated with MATLAB CODER option. The generated $\mathrm{C}$ code of the UKF algorithm is then downloaded to the $150-\mathrm{MHz}$ DSP TMS320F28335 from Texas Instruments 


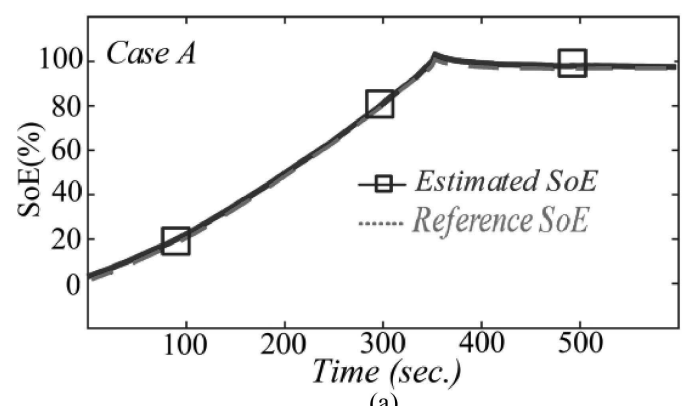

(a)

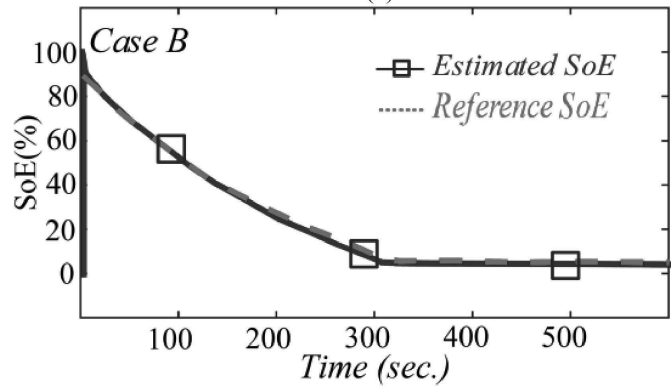

(b)

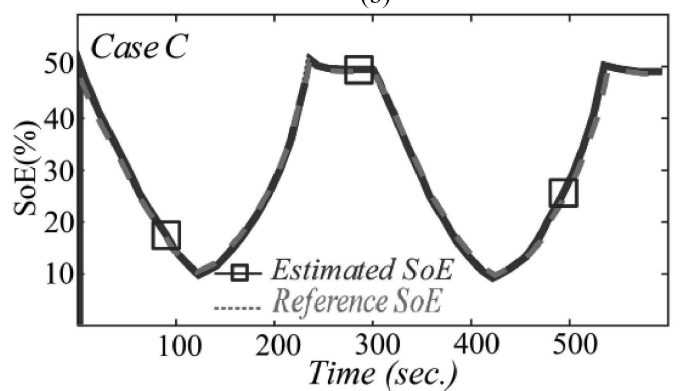

(c)

Fig. 8. Estimated SoE with the proposed UKF-based approach. (a) Case A. (b) Case B. (c) Case C.

using Code Composer Studio 6.2.0 software. A pre-recorded dataset, which includes the supercapacitor voltage and current signals, is imported to MATLAB in a host computer. The current and voltage waveforms are contaminated with random Gaussian White noise with SNR of $30 \mathrm{~dB}$ to effectively mimic the reallife conditions. The obtained voltage and current data are then sent from the host computer to the DSP in an online manner using the PCI-1712 data-acquisition card. In the meanwhile, analog low-pass antialiasing filters of order two with a cut-off frequency of $2 \mathrm{kHz}$ are used. Upon receiving each data sample by DSP, the supercapacitor SoH and SoE are estimated with the proposed algorithm. The results are finally sent back to the host computer for monitoring and controlling purposes (to synchronize the whole HIL process). The required memory for the proposed UKF-based estimator is about $27 \mathrm{kbytes}$, which is far lower than the memory of TMS320F28335 $(256 \mathrm{~K} \times 16$ flash memory). Furthermore, the maximum run-time of the algorithm for the estimation is about $0.091 \mathrm{~ms}$. A sampling frequency of 1 $\mathrm{kHz}$ is selected for the algorithm (which is sufficiently good for state estimation in vehicular applications) and thus, each iteration must be accomplished within $1 \mathrm{~ms}$. Therefore, only $9.1 \%$ of the processor resources will be used by the proposed algorithm
TABLE II

State Estimation ERroRs of SoE For DiffERENT Scenarios

\begin{tabular}{ccccc}
\hline Scenario & Case $\boldsymbol{A}$ & Case B & Case $\boldsymbol{C}$ & Case D \\
\hline \hline SoE error $\%$ & $0.473 \%$ & $0.512 \%$ & $0.621 \%$ & $0.813 \%$ \\
\hline
\end{tabular}

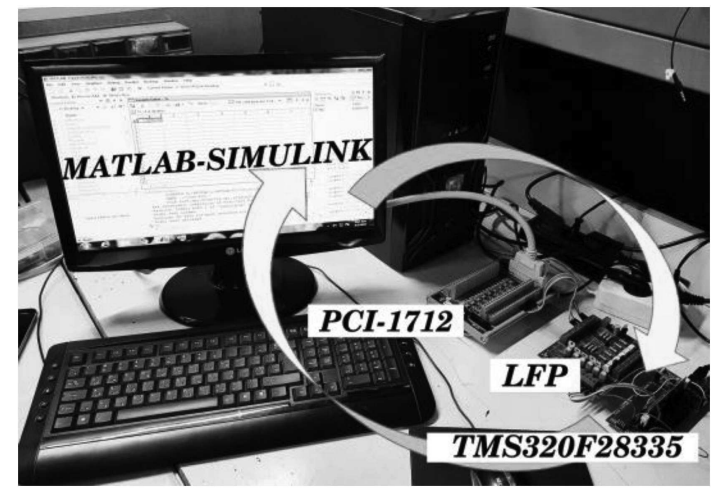

Fig. 9. Photo of the testbench for HIL tests.

TABLE III

Comparison Between the Proposed Method AND KF-BASED ALGORITHMS

\begin{tabular}{cccc}
\hline Method & $\begin{array}{c}\text { SoH } \\
\text { Accuracy }\end{array}$ & $\begin{array}{c}\text { SoE } \\
\text { Accuracy }\end{array}$ & CPU usage \\
\hline \hline KF-based [16] & $\approx 98 \%$ & $\approx 99 \%$ & $5.2 \%$ \\
\hline \hline EKF-based [17] & $\approx 95 \%$ & $\times$ & $13.5 \%$ \\
\hline \hline UKF-based (proposed) & $\approx 99.58 \%$ & $\approx 99.4 \%$ & $9.1 \%$ \\
\hline
\end{tabular}

$\times$ denotes that the estimation is not considered. All algorithms are tested using TMS320F28335 with a sampling frequency of $1 \mathrm{kHz}$.

and there will be no bottleneck for real-time implementation of the algorithm.

\section{Comparison With Similar Methods}

In this section, a comparison between the proposed method with other KF-based approaches in terms of accuracy and computational complexity is presented. The results are summarized in Table III. The comparison reveals that the proposed UKFbased approach provides better accuracy for supercapacitor $\mathrm{SoH}$ and SoE estimation in comparison with KF-based and EKFbased methods. In addition, the computational burden of the proposed approach is lower than the EKF method, though the KF-based method still has the lowest computational complexity.

\section{CONCLUSION}

A state estimation approach based on the UKF algorithm for joint estimation of the supercapacitor $\mathrm{SoH}$ and SoE was proposed in this article. A first-order equivalent circuit model that takes into account the self-discharge and capacitance variation effects was developed. The supercapacitor model parameters and its internal voltage were augmented in one state-space model for concurrent estimation of SoH and SoE using the UKF algorithm. Unlike the KF and EKF algorithms which involve using a linearized supercapacitor model, the proposed UKF-based 
TABLE IV

Rated Values of The Under-Study Supercapacitor Cell

\begin{tabular}{cc}
\hline Parameter & Value \\
\hline \hline Rated capacitance & $350 \mathrm{~F}$ \\
Rated voltage & $2.7 \mathrm{~V}$ \\
Absolute maximum voltage & $2.85 \mathrm{~V}$ \\
Equivalent series resistance & $3.2 \mathrm{~m} \Omega$ \\
Leakage current $\left(\right.$ at $\left.T=25^{\circ} \mathrm{C}\right)$ & $0.3 \mathrm{~mA}$ \\
Specific energy & $5.9 \mathrm{Wh} / \mathrm{kg}$ \\
Maximum continuous current & $21 \mathrm{~A}$ \\
\hline
\end{tabular}

method achieved higher accuracy due to the use of nonlinear supercapacitor dynamics. Moreover, it effectively dealt with the issues relevant to the measurement errors and modeling uncertainties with the involvement of covariance matrices of the measurement and process noise. While the proposed approach achieved higher accuracy than the state-of-the-art, its computational burden was remarkably low, which maked it a good candidate for real-time vehicular applications.

\section{APPENDIX}

The supercapacitor cell used for the study is a Maxwell $350 \mathrm{~F}$ radial $\mathrm{D}-\mathrm{Cell}$. The rated values of the cell are provided in Table IV.

\section{REFERENCES}

[1] F. Naseri, E. Farjah, and T. Ghanbari, "An efficient regenerative braking system based on battery/supercapacitor for electric, hybrid, and plug-in hybrid electric vehicles with BLDC motor," IEEE Trans. Veh. Technol., vol. 66, no. 5, pp. 3724-3738, May 2017.

[2] A. Alessandrini et al., "Design of a hybrid storage for road public transportation systems," in Proc. Int. Conf. IFToMM ITALY, 2018, pp. 149-157.

[3] J. Cao and A. Emadi, "A new battery/ultracapacitor hybrid energy storage system for electric, hybrid, and plug-in hybrid electric vehicles," IEEE Trans. Power Electron., vol. 27, no. 1, pp. 122-132, Jan. 2012.

[4] S. M. Lukic, J. Cao, R. C. Bansal, F. Rodriguez, and A. Emadi, "Energy storage systems for automotive applications," IEEE Trans. Ind. Electron., vol. 55, no. 6, pp. 2258-2267, Jun. 2008.

[5] R. Kötz, P. W. Ruch, and D. Cericola, "Aging and failure mode of electrochemical double layer capacitors during accelerated constant load tests," J. Power Sources, vol. 195, no. 3, pp. 923-928, 2010.

[6] S. Buller, E. Karden, D. Kok, and R. W. De Doncker, "Modeling the dynamic behavior of supercapacitors using impedance spectroscopy," IEEE Ind. Appl. Conf., vol. 4, no. 6, pp. 2500-2504, Nov./Dec. 2001.

[7] P. Kreczanik, P. Venet, A. Hijazi, and G. Clerc, "Study of supercapacitor aging and lifetime estimation according to voltage, temperature, and RMS current," IEEE Trans. Ind. Electron., vol. 61, no. 9, pp. 4895-4902, Sep. 2014.

[8] A. Soualhi et al., "Supercapacitors ageing prediction by neural networks," in Proc. IEEE Ind. Electron. Soc. Conf., 2013, pp. 6812-6818.

[9] A. Oukaour et al., "Supercapacitors aging diagnosis using least square algorithm," Microelectron. Rel., vol. 53, no. 9, pp. 1638-1642, 2013.
[10] N. Reichbach and A. Kuperman, "Recursive-least-squares-based real-time estimation of supercapacitor parameters," IEEE Trans. Energy Convers., vol. 31, no. 2, pp. 810-812, Jun. 2016.

[11] N. Reichbach, S. Kolesnik, and A. Kuperman, "Real-time state-of-energy estimation of supercapacitor-based energy storage," in Proc. IEEE EastWest Des. Test Symp., 2015, pp. 1-4.

[12] A. Eddahech, M. Ayadi, O. Briat, and J. M. Vinassa, "Online parameter identification for real-time supercapacitor performance estimation in automotive applications," Int. J. Elect. Power Energy Syst., vol. 51, pp. 162-167, 2013.

[13] F. Naseri, E. Farjah, M. Allahbakhshi, and Z. Kazemi, "Online condition monitoring and fault detection of large supercapacitor banks in electric vehicle applications," IET Elect. Syst. Transp., vol. 7, no. 4, pp. 318-326, 2017.

[14] M. Ceraolo, G. Lutzemberger, and D. Poli, "State-of-charge evaluation of supercapacitors," J. Energy Storage, vol. 11, pp. 211-218, 2017.

[15] Y. Zhou, Z. Huang, H. Li, J. Peng, W. Liu, and H. Liao, “A generalized extended state observer for supercapacitor state of energy estimation with online identified model," IEEE Access, vol. 6, pp. 27706-27716, 2018.

[16] A. Nadeau, G. Sharma, and T. Soyata, "State-of-charge estimation for supercapacitors: A Kalman filtering formulation," in Proc. IEEE Int. Conf. Acoust., Speech, Signal Process., 2014, pp. 2194-2198.

[17] A. El Mejdoubi, A. Oukaour, H. Chaoui, Y. Slamani, J. Sabor, and H. Gualous, "Online supercapacitor diagnosis for electric vehicle applications," IEEE Trans. Veh. Technol., vol. 65, no. 6, pp. 4241-4252, Jun. 2016.

[18] A. Nadeau, M. Hassanalieragh, G. Sharma, and T. Soyata, "Energy awareness for supercapacitors using Kalman filter state-of-charge tracking," J. Power Sources, vol. 296, pp. 383-391, 2015.

[19] E. Locorotondo, L. Pugi, L. Berzi, M. Pierini, and A. Pretto, "Online state of health estimation of lithium-ion batteries based on improved amperecount method," in Proc. IEEE Int. Conf. Environ. Elect. Eng. 2018 IEEE Ind. Commercial Power Syst. Europe, 2018, pp. 1-6.

[20] E. Locorotondo, L. Pugi, L. Berzi, M. Pierini, and G. Lutzemberger, "Online identification of Thevenin equivalent circuit model parameters and estimation state of charge of lithium-ion batteries," in Proc. IEEE Int. Conf. Environ. Elect. Eng. , IEEE Ind. Commercial Power Syst. Europe, 2018, pp. 1-6.

[21] W. Li, G. Joós, and J. Bélanger, "Real-time simulation of a wind turbine generator coupled with a battery supercapacitor energy storage system," IEEE Trans. Ind. Electron., vol. 57, no. 4, pp. 1137-1145, Apr. 2010.

[22] N. Kularatna, J. Fernando, A. Pandey, and S. James, "Surge capability testing of supercapacitor families using a lightning surge simulator," IEEE Trans. Ind. Electron., vol. 58, no. 10, pp. 4942-4949, Oct. 2011.

[23] Y. Y. Liu, J. J. Slotine, and A. L. Barabási, "Observability of complex systems," Proc. Nat. Acad. Sci., vol. 110, no. 7, pp. 2460-2465, 2013.

[24] F. Naseri, Z. Kazemi, M. M. Arefi, and E. Farjah, "Fast discrimination of transformer magnetizing current from internal faults: An extended Kalman filter-based approach," IEEE Trans. Power Del., vol. 33, no. 1, pp. 110 118, Feb. 2018

[25] F. Naseri, Z. Kazemi, E. Farjah, and T. Ghanbari, "Fast detection and compensation of current transformer saturation using extended Kalman filter," IEEE Trans. Power Del., vol. 34, no. 3, pp. 1087-1097, Jun. 2019.

[26] T. Ghanbari, E. Farjah, F. Naseri, N. Tashakor, H. Givi, and R. Khayam, "Solid-state capacitor switching transient limiter based on Kalman filter algorithm for mitigation of capacitor bank switching transients," Renewable Sustain. Energy Rev., vol. 90, pp. 1069-1081, 2018.

[27] D. Simon, Optimal State Estimation: Kalman, H Infinity, and Nonlinear Approaches. New York, NY, USA: Wiley, 2006. 